\title{
Birth of Piglets through the Non-Surgical Transfer of Blastocysts Produced In Vitro
}

\author{
Chie SUZUKI ${ }^{1)}$, Shokichi IWAMURA ${ }^{1)}$ and Koji YOSHIOKA ${ }^{1)}$ \\ ${ }^{1)}$ Department of Production Diseases, National Institute of Animal Health, 3-1-5 Kannondai, \\ Tsukuba, Ibaraki 305-0856, Japan
}

\begin{abstract}
In this study, we attempted to produce piglets by non-surgically transferring blastocysts produced in vitro, using a flexible catheter as the transfer instrument. Cumulus-oocyte complexes (COCs) were aspirated from the follicles of ovaries obtained at a local slaughterhouse. They were then matured in modified North Carolina State University (NCSU)-37 medium for $44-46 \mathrm{~h}$ and fertilized in porcine gamete medium (PGM). Ten hours after in vitro fertilization (IVF), presumptive zygotes were removed from the cumulus cells and cultured in porcine zygote medium (PZM)-5. Blastocysts were cultured for five days after IVF and, using a catheter for deep intrauterine insemination without sedation, they were transcervically transferred into the uterine horn of six recipients (45-50 blastocysts/recipients) whose estrous cycles were synchronized, at 5 days after human chorionic gonadotropin (hCG) injection. Of the six recipients, one sow became pregnant and farrowed seven piglets (four live piglets) 119 days after hCG injection. The body weight at birth of the newborns ranged from 0.8 to $1.4 \mathrm{~kg}$. These results indicate that it is possible to obtain piglets by transcervically transferring blastocysts produced by IVF and in vitro cultures in chemically defined media.
\end{abstract}

Key words: In vitro-produced blastocysts, Non-surgical embryo transfer, Production of piglets

(J. Reprod. Dev. 50: 487-491, 2004)

$\mathbf{P}$ orcine embryo transfer could contribute to the controlling mechanisms of animal reproduction, including transgenesis and cloning [1]. In pigs, embryo transfer techniques are important not only for conducting genetic improvements and maintaining superior genotypes but also for the clearance of chronic infectious diseases, such as Aujeszky's disease, porcine reproductive and respiratory syndrome, and mycoplasmal pneumonia of swine. It is difficult to collect and transfer pig embryos using non-surgical techniques because of the anatomical complexity of the cervix and uterus. Thus, pig embryos are generally collected through surgery or after the slaughter of a donor, and transferred surgically into recipients [2].

Accepted for publication: April 7, 2004

Correspondence: C. Suzuki (e-mail: schie@affrc.go.jp)
Recently, Yoshioka et al. developed a porcine in vitro production (IVP) system using chemically defined media [3,4]. Blastocysts produced with this system can effectively develop to full-term after surgical transfer. Non-surgical embryo transfer has the advantage that surgical facilities and techniques are not required. There are some reports that document the production of piglets by transfer of embryos derived in vivo into the uterine body or uterine horn using a catheter for artificial insemination or a specially designed flexible instrument [5-11]. However, there are currently no reports that document the production of piglets by non-surgical transfer of IVP embryos. This study was designed to examine the developmental competence of IVP embryos after transcervical transfer without surgical procedures or anesthesia using a catheter for deep intrauterine insemination 


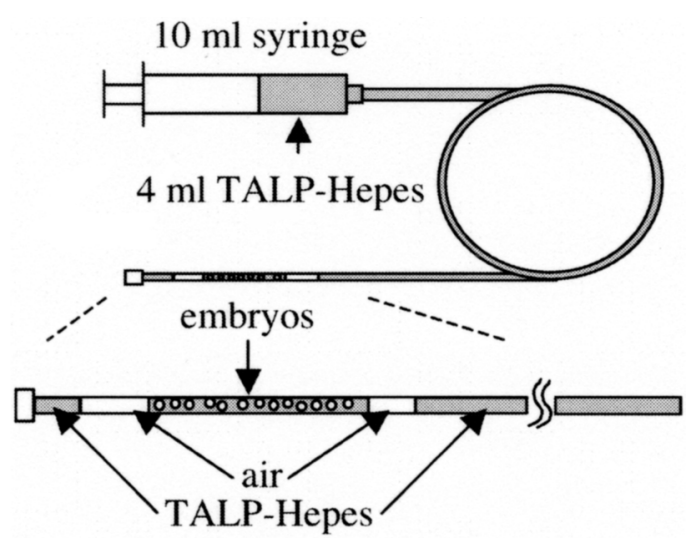

Fig. 1. Diagram showing the set-up of the transferred embryos in the extension tube. Embryos were placed into the end of an extension tube attached to a $10-\mathrm{ml}$ syringe. The tube and syringe were filled with approximately $6 \mathrm{ml}$ of TALP-Hepes as a transfer medium.

as the transfer instrument.

\section{Materials and Methods}

\section{IVP of porcine embryos}

In vitro maturation (IVM) and in vitro fertilization (IVF) were conducted as described previously [3]. Briefly, the COCs were aspirated from medium sized follicles (3-6 $\mathrm{mm}$ in diameter) of ovaries collected from prepubertal gilts in a slaughterhouse. The COCs were then washed twice with Hepes-buffered Tyrode medium (TALPHepes), and twice with modified North Carolina State University (NCSU)-37 medium supplemented with $10 \%(\mathrm{v} / \mathrm{v})$ porcine follicular fluid, $0.6 \mathrm{mM}$ cysteine, $1 \mathrm{mM}$ dibutyryl cAMP (dbcAMP), $10 \mathrm{IU} /$ ml hCG (Gonatropin; Teikoku Hormone MFG., Tokyo, Japan) $10 \mathrm{IU} / \mathrm{ml}$ eCG (Serotropin; Teikoku Hormone MFG.), and $50 \mu \mathrm{g} / \mathrm{ml}$ gentamicin sulfate. They were then cultured for $20-22 \mathrm{~h}$ in the same medium and for a further $24 \mathrm{~h}$ in the same medium without the hormone or dbcAMP.

IVF was performed using the same batch of frozen-thawed ejaculated semen. The IVF medium was porcine gamete medium containing $2.5 \mathrm{mM}$ theopylline, $1 \mu \mathrm{M}$ adenosine and $0.2 \mu \mathrm{M}$ cysteine (PGM-tac [3]). Frozen-thawed semen layered on Percoll gradient $(90 \%$ and $45 \%)$ was centrifuged at $700 \times \mathrm{g}$ for $20 \mathrm{~min}$ and then washed with PGM-tac

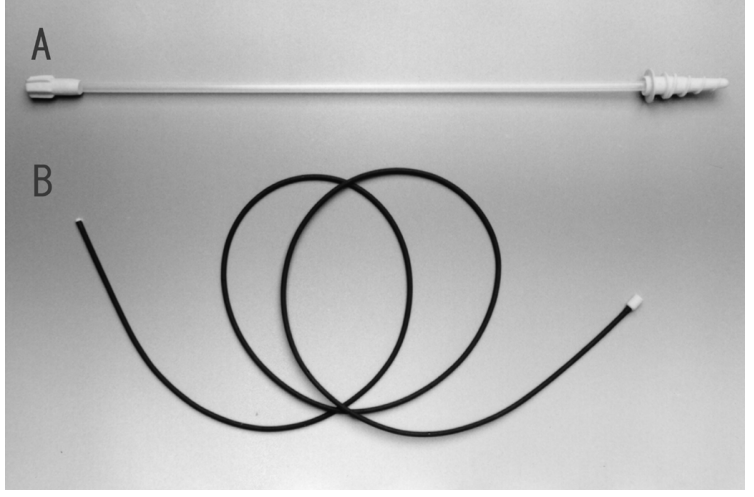

Fig. 2. Catheters used for non-surgical pig embryo transfer. The AI catheter (A) and the flexible catheter (B) were used as a guide to the external uterine orifice, and for deep intrauterine embryo transfer, respectively.

by centrifugation at $500 \times \mathrm{g}$ for $5 \mathrm{~min}$. After IVM, the COCs were co-incubated for $10 \mathrm{~h}$ with the spermatozoa at a concentration of $2 \times 10^{6}$ spermatozoa/ml in $100 \mu \mathrm{l}$ droplets of PGM-tac. Mean $( \pm S D)$ proportions of oocytes penetrated and fertilized normally in the present study were $75.2 \pm$ $16.8 \%$ and $47.4 \pm 13.8 \%$, respectively. They were then transferred to a $15-\mathrm{ml}$ conical tube with $1 \mathrm{ml}$ TALP-Hepes and the cumulus cells were removed from the oocytes by vortexing for $4 \mathrm{~min}$. Presumptive zygotes were washed three times with TALP-Hepes and once with porcine zygote medium (PZM)-5 [12], in which the glutamine concentration was modified from $1 \mathrm{mM}$ to $2 \mathrm{mM}$ in PZM-4 [4]. Presumptive zygotes were cultured in PZM-5 for five days. IVM, IVF and IVC were performed at $39 \mathrm{C}$ in a humidified atmosphere containing $5 \% \mathrm{CO}_{2}, 5 \% \mathrm{O}_{2}$, and $90 \% \mathrm{~N}_{2}$.

\section{Non-surgical embryo transfer}

Six sows (Large White or Landrace $\times$ Large White; 12-15 months old; 153-187 kg body weight) were used as the recipients for non-surgical embryo transfer. All animal-related procedures followed in this study were approved by the Institutional Care and Use Committee for Laboratory Animals of the National Institute of Animal Health (protocol no. 398). Luteolysis of the recipients was induced by intramuscular injections of prostaglandin (PG) $\mathrm{F}_{2 \alpha}$ (Panacelan Hi, Daiichi Pharmaceutical Co. Ltd., 
Tokyo, Japan) as $15 \mathrm{mg}$ of dinoprost twice daily for 3 days during the functional luteal phase. Ovulation was induced with intramuscular injections of $1500 \mathrm{IU}$ hCG $72 \mathrm{~h}$ after the fifth $\mathrm{PGF}_{2 \alpha}$ treatment [3]. Blastocysts cultured for 5 days in PZM-5 were washed twice with TALP-Hepes and placed (45-50 embryos) in the end of a polyvinyl chloride tube $\left(S_{A F E E D}{ }^{\circledR}\right.$ Extension Tube, SFET2527, Terumo, Tokyo, Japan), as shown in Fig. 1. The tube including the embryos was transferred to the pig house in a heat-insulating box at $37 \mathrm{C}$. Embryo transfer was performed within $1 \mathrm{~h}$ on each sow without the use of tranquilizers or anesthesia. A flexible catheter (FIREFLEX ${ }^{\circledR}$, Magapor, Zaragoza, Spain) was used for the embryo transfer. The perineal area of each recipient was disinfected with benzalkonium chloride and the vulva was cleaned with alcohol cotton. A commercial artificial insemination (AI) catheter covered with a vinyl sheath was introduced into the cervical opening through the vagina using a colposcope. The vinyl sheath was removed after reaching the cervical opening. The AI catheter was then inserted into the cervix by turning it counter-clockwise until it was firmly locked in place. The flexible catheter was inserted 100-120 cm through the AI catheter after the inside of the flexible catheter was rinsed with 2-3 ml TALP-Hepes. The tube including the embryos was attached to the end of this flexible catheter and, therefore, along with the $6 \mathrm{ml}$ TALPHepes the embryos were infused into the uterus (45-50 embryos/animal).

Pregnancy diagnosis was carried out by ultrasonography 21 days after hCG injection. Animals that were diagnosed as pregnant were allowed to term and the gestation length, litter size and body weight of the piglets at birth were examined.

\section{Result and Discussion}

A total of 948 presumptive zygotes were cultured in PZM-5 following IVF. Two hundred eightyseven embryos (30.2\%) developed to the blastocyst stage on Day 5. Of a total of 287 blastocysts, 284 embryos were transferred into six recipients. The result of embryo transfer for each recipient is presented in Table 1. Of the six recipients, only one achieved pregnancy and farrowed seven piglets; of these four were alive. No clinical abnormalities were observed in the piglets that were born. The gestation length and body weight of the piglets were within the normal range.

This is the first report to document the birth of piglets by non-surgical transfer of IVP blastocysts. Several studies have reported piglet production by surgical embryo transfer using porcine IVP blastocysts [3, 13, 14]. Yoshioka et al. [3] revealed that $25-30 \%$ of zygotes cultured in PZM-4 developed to blastocysts, and after surgical transfer of 90 blastocysts into 4 recipients, all were able to farrow a total of 21 piglets. Furthermore, in a previous study, the authors reported that PZM-5, in which the glutamine concentration was altered from $1 \mathrm{mM}$ to $2 \mathrm{mM}$ in PZM-4, improved blastocyst yield and the total number of cells in these blastocysts (31\% and 42.9 cells, respectively) [12]. Although, in this study, the blastocysts were produced in a culture with PZM-5, only one recipient of the six sows impregnated farrowed piglets. To increase the conception rate with non-

Table 1. Result of transcervical embryo transfer in pigs

\begin{tabular}{|c|c|c|c|c|c|}
\hline Recipient & $\begin{array}{l}\text { No. of embryos } \\
\text { transferred }\end{array}$ & Pregnancy & $\begin{array}{c}\text { Gestation length } \\
\text { (days) }^{\mathrm{a}}\end{array}$ & $\begin{array}{c}\text { Total no. of } \\
\text { piglets born } \\
\text { (No. of live piglets) }\end{array}$ & $\begin{array}{l}\text { Average body weight of } \\
\text { piglets at birth }{ }^{\mathrm{b}} \text { (of living } \\
\text { piglets) (kg) [range] }\end{array}$ \\
\hline A & 47 & no & - & - & - \\
\hline B & 47 & yes & 117 & $7(4)$ & $\begin{array}{c}1.2 \pm 0.2(1.3 \pm 0.1) \\
{[0.8-1.4(1.1-1.4)]}\end{array}$ \\
\hline $\mathrm{C}$ & 50 & no & - & - & - \\
\hline $\mathrm{D}$ & 50 & no & - & - & - \\
\hline $\mathrm{E}$ & 45 & no & - & - & - \\
\hline $\mathrm{F}$ & 45 & no & - & - & - \\
\hline
\end{tabular}

${ }^{\text {aDay }} 0=2$ days after hCG injection.

${ }^{\mathrm{b}}$ Mean \pm SD. 
surgical transfer of IVP embryos, further improvements in the catheter technique and handling of the embryos are needed.

Several reports have documented the success of non-surgical embryo transfer using porcine embryos developed in vivo [5-11]. In general, the farrowing rate of non-surgical embryo transfer (9$64 \%$ ) [5-11] is lower than that of surgical embryo transfer (17-100\%) [15]. In the reports on nonsurgical embryo transfer, embryos were deposited in the uterine horn near the uterine body or the cervix. The location of the tip of catheter is important for the success of conception with embryo transfer. Wallenhorst et al. [16] revealed that by transferring embryos near the tip of the uterine horn they could achieve a high rate of embryo survival. In this study we attempted to transfer embryos deep into the uterine horn. The flexible catheter used in this study can be inserted deep into the uterine horn through the cervix for AI in estrous sows [17]. In contrast, there are some reports that claim it is not easy to insert a catheter into the uterine horn during the luteal phase because of cervix restriction $[2,6]$. In some cases during the present study, the tip of the catheter might not have reached deep into the uterine horn. The location of the tip of the inserted catheter needs to be confirmed in future trials.

Transferring embryos with large amounts of medium might result in a backward flow of the embryos within the medium towards the vagina $[18,19]$. In the early stages of pregnancy, substantial amounts of fluid are typically not found in the uterus, and these non-physiological conditions might affect the survival rate of the embryos [18, 19]. Yonemura et al. [20] reported that the pregnancy rate and number of fetuses transferred with $15 \mathrm{ml}$ of medium using the AI catheter into the cervix, was higher than that of embryos transferred with $30 \mathrm{ml}$ of medium. Although the embryos were transferred with 6-7 $\mathrm{ml}$ of medium in our study, only a minimal amount of medium should be used to flush the embryos from the catheter to increase the success of pregnancy after embryo transfer. Thus, the amount of medium used in the flexible catheter will need to be investigated in the future.

In porcine embryo transfer, when the estrous cycle of the recipients was asynchronous to that of the donors with a 2-day delay, the pregnancy rate appeared to be high [2]. In this study, Day-5 blastocysts were transferred after IVF into the recipients 5 days after hCG treatment, which is similar to the schedule used with 2-day delay, resulting in the birth of piglets. The development of IVP embryos is generally slower than that of embryos developed in vivo, and the cell number of IVP blastocysts (37-46 cells at 5 days after IVF [3, 12]) is smaller than that of blastocysts developed in vivo (62 cells at 4 days after ovulation [4]). To increase the farrowing rate, the effects of IVP embryo age and the estrous cycle of the recipients on conception after embryo transfer will also need to be examined.

In this study, piglets were born after non-surgical IVP embryo transfer. The production of piglets by non-surgical IVP embryo transfer is more economical than by the surgical transfer of embryos developed in vivo. To increase the farrowing rate and number of piglets born after non-surgical IVP embryo transfer, further methodological improvements are necessary.

\section{Acknowledgments}

This work was supported by a grant from the Ministry of Agriculture, Forestry and Fisheries of Japan. The authors are grateful to Dr. Itoh of Azabu University and the staff of Nagano Prefecture Livestock Experimental Station for providing frozen boar semen.

\section{References}

1. Wall RJ. Transgenic livestock: progress and prospects for the future. Theriogenology 1996; 45: 5768.

2. Polge C. Embryo transplantation and preservation. In: Cole DJA, Foxcroft GR (eds.), Control of Pig Reproduction. London: Butterworth Scientific; 1982:
279-291.

3. Yoshioka K, Suzuki C, Itoh S, Kikuchi K, Iwamura S, Rodriguez-Martinez H. Production of piglets derived from in vitro produced blastocysts fertilized and cultured in chemically defined media: effects of theophylline, adenosine and cysteine during in vitro 
fertilization. Biol Reprod 2003; 69: 2092-2099.

4. Yoshioka K, Suzuki C, Tanaka A, Anas IMK, Iwamura S. Birth of piglets derived from porcine zygotes cultured in a chemically defined medium. Biol Reprod 2002; 66: 112-119.

5. Polge C, Day BN. Pregnancy following non-surgical egg transfer in pigs. Vet Rec 1968; 82: 712.

6. Reichenbach HD, Modl J, Brem G. Piglets born after transcervical transfer of embryos into recipient gilts. Vet Rec 1993; 133: 36-39.

7. Galvin JM, Killian DB, Stewart ANV. A procedure for successful non-surgical embryo transfer in swine. Theriogenology 1994; 41: 1279-1289.

8. Li J, Rieke A, Day BN, Prather RS. Technical note: porcine non-surgical embryo transfer. J Anim Sci 1996; 74: 2263-2268.

9. Hazeleger W, Kemp B. Farrowing rate and litter size after transcervical embryo transfer in sows. Reprod Dom Anim 1994; 29: 481-487.

10. Yonemura I, Fujino $\mathbf{Y}$, Irie S, Miura $\mathbf{Y}$. Transcervical transfer of porcine embryos under practical conditions. J Reprod Dev 1996; 42: 89-94.

11. Hazeleger W, Bouwman EG, Noordhuizen JPTM, Kemp B. Effect of superovulation induction on embryonic development on Day 5 and subsequent development and survival after non-surgical embryo transfer. Theriogenology 2000; 53: 1063-1070.

12. Suzuki C, Yoshioka K, Iwamura S. Effects of amino acids and BSA on the development of in vitro produced porcine embryos. In: Program of the 95th
Annual Meeting of the Japanese Society of Animal Reproduction; 2002; Iwate, Japan (In Japanese).

13. Kikuchi K, Onishi A, Kashiwazaki N, Iwamoto M, Noguchi J, Kaneko H, Akita T, Nagai T. Successful piglet production after transfer of blastocysts produced by a modified in vitro system. Biol Reprod 2002; 66: 1033-1041.

14. Marchal R, Feugang JM, Perreau C, Venturi E, Terqui M, Mermillo P. Meiotic and developmental competence of prepubertal and adult swine oocytes. Theriogenology 2001; 56: 17-29.

15. Pope WF, Maurer RR, Stormshak F. Survival of porcine embryos after asynchronous transfer. Proc Soc Exp Biol Med 1982; 171: 179-183.

16. Wallenhorst $\mathbf{S}$, Holtz $\mathbf{W}$. Transfer of pig embryos to different uterine sites. J Anim Sci 1999; 77: 23272329.

17. Martinez EA, Vazquez JM, Roca J, Lucas X, Gil MA, Parrilla I, Vazquez JL, Day BN. Successful non-surgical deep intrauterine insemination with small numbers of spermatozoa in sows. Reproduction 2001; 122: 289-296.

18. Hazeleger W, Kemp B. State of the art in pig embryo transfer. Theriogenlogy 1999; 51: 81-90.

19. Hazeleger W, Kemp B. Recent developments in pig embryo transfer. Theriogenology 2001; 56: 1321-1331.

20. Yonemura I, Miyamoto $\mathbf{K}$, Nishida $\mathbf{M}$. Nonsurgical transfer of porcine embryos. Theriogenology 2003; 59: 378 (abstract). 